\title{
BMJ Open Treatment of early-stage breast cancer with percutaneous thermal ablation, an open-label randomised phase 2 screening trial: rationale and design of the THERMAC trial
}

To cite: van de Voort EMF Struik GM, Koppert LB, et al. Treatment of early-stage breast cancer with percutaneous thermal ablation, an open-label randomised phase 2 screening trial: rationale and design of the THERMAC trial. BMJ Open 2021;11:e052992. doi:10.1136/ bmjopen-2021-052992

- Prepublication history and additional supplemental material for this paper are available online. To view these files, please visit the journal online. (http://dx.doi.org/10.1136/ bmjopen-2021-052992).

Received 05 May 2021 Accepted 28 July 2021

Check for updates

(C) Author(s) (or their employer(s)) 2021. Re-use permitted under CC BY-NC. No commercial re-use. See rights and permissions. Published by BMJ.

For numbered affiliations see end of article.

Correspondence to Drs Elles M F van de Voort; e.vandevoort@erasmusmc.nl

\section{ABSTRACT}

Introduction Breast cancer is the most frequently diagnosed malignancy worldwide but almost half of the patients have an excellent prognosis with a 5-year survival rate of $98 \%-99 \%$. These patients could potentially be treated with thermal ablation to avoid surgical excision, reduce treatment-related morbidity and increase patients' quality of life without jeopardising treatment effectiveness. Previous studies showed highest complete ablation rates for radiofrequency, microwave and cryoablation. However, due to heterogeneity among studies, it is unknown which of these three techniques should be selected for a phase 3 comparative study.

Methods and analysis The aim of this phase 2 screening trial is to determine the efficacy rate of radiofrequency, microwave and cryoablation with the intention to select one treatment for further testing in a phase 3 trial.

Additionally, exploratory data are obtained for the phase 3 trial. The design is a multicentre open-label randomised phase 2 screening trial. Patients with unifocal, invasive breast cancer with a maximum diameter of $2 \mathrm{~cm}$ without lymph node or distant metastases are included. Triple negative, Bloom-Richardson grade 3 tumours and patients with an indication for neoadjuvant chemotherapy will be excluded. Included patients will be allocated to receive one of the three thermal ablation techniques. Three months later surgical excision will be performed to determine the efficacy of thermal ablation. Treatment efficacy in terms of complete ablation rate will be assessed with CK 8/18 and H\&E staining. Secondary outcomes include feasibility of the techniques in an outpatient setting, accuracy of MRI for complete ablation, patient satisfaction, adverse events, side effects, cosmetic outcome, system usability and immune response.

Ethics and dissemination This study protocol was approved by Medical Research Ethics Committee of the Erasmus Medical Center, Rotterdam, the Netherlands. Study results will be submitted for publication in peerreviewed journals.

Trial registration number NL9205 (www.trialregister.nl); Pre-results.
Strengths and limitations of this study

This is the first study in which the three most promising thermal ablation techniques will be tested according to a uniform study protocol among patients with early-stage breast cancer.

- In this protocol, there is no direct benefit for patients to participate as surgical excision is still needed, which could impact the inclusion rate.

- The prolonged interval between thermal ablation and surgical excision will allow adequate assessment of complete ablation on MRI and pathology taking both direct and delayed effects of thermal ablation into account.

- Long-term follow-up will provide data on the cosmetic outcome of thermal ablation, in combination with surgical excision and radiotherapy which has not been described before.

\section{INTRODUCTION}

Breast cancer is the most frequently diagnosed malignancy worldwide. In the Netherlands, almost 15000 patients were diagnosed with breast cancer in 2019. ${ }^{1}$ Almost half of all detected tumours are smaller than $2 \mathrm{~cm}$ and without lymph node or distant metastases due to improvements in screening. With current therapy, these patients with an early-stage breast cancer have an excellent prognosis; the 5-year survival rate is $98 \%-99 \% .{ }^{23}$ However, early detection may have also resulted in overtreatment. ${ }^{4}$ It is therefore important to focus research on treatments that reduce treatment-related morbidity and increase patients' quality of life.

Percutaneous thermal ablation is a minimally invasive treatment that has the potential to replace surgery for early-stage breast 
cancer. It is already successfully being used for other focal malignancies. ${ }^{5}$ Various thermal ablation techniques for the treatment of primary breast cancer have been investigated. According to the latest meta-analyses, radiofrequency ablation (RFA), cryoablation (CA) and microwave ablation (MWA) have the highest efficacy in terms of the proportion of patients in which complete ablation is seen after thermal ablation. In tumours $\leq 2 \mathrm{~cm}$ complete ablation rates of $82 \%-92 \%, 83 \%-90 \%$ and $74 \%-85 \%$ were found, respectively. ${ }^{6-8}$ High complete ablation rates $(91 \%)$ are reported in the latest ongoing cohort studies, in which no subsequent surgical resection was performed. ${ }^{9}$ Preliminary reported local recurrence rates are $0 \%-1 \%$ in these studies after a maximum follow-up of 5 years. ${ }^{9}{ }^{10}$ Over time, devices have improved, operators have become more experienced, and patient selection has changed, resulting in a large heterogeneity across studies. ${ }^{6-8}$ Therefore, the actual complete ablation rates for RFA, MWA and CA with current devices and optimal patient selection are unknown. It remains unclear which of these techniques is optimal for a comparative phase three study.

Thermal ablation causes both immediate and delayed effects, because the area of cell death will expand over time. ${ }^{11}{ }^{12}$ Delayed cell death occurs due to vascular thrombosis, resulting in progressive failure of the microcirculation and ultimately vascular stasis, tissue ischaemia or reperfusion injury. ${ }^{13} 14$ Additionally, it is postulated that thermal ablation will activate the immune system and thereby eradicate residual tumour cells over time. ${ }^{14-18}$ due to these delayed effects, it is likely that complete ablation rates increase when the interval between thermal ablation and evaluation of the specimen is prolonged.

To safely avoid surgery after thermal ablation, an important challenge will be to reliably evaluate complete ablation. MRI seems promising to evaluate complete ablation reliably, but the results in literature remain ambiguous. On one hand, repeating postablation MRI may result in higher specificity and improved discrimination between reactive granulation tissue and residual disease. ${ }^{19}$ On the other hand, ductal carcinoma in situ (DCIS) or foci outside of the primary tumour could still be missed on postablation MRI. ${ }^{20} 21$ The method for determining complete ablation should at least have sufficient sensitivity, to maintain the current low local recurrence rate of patients with early-stage breast cancer.

Other important aspects such as cosmetic outcome, initiation of an immune response, and quality of life are currently under-reported in literature. For example, results of cosmetic outcome and patient satisfaction are sparsely reported and include mainly short-term outcomes without the use of validated questionnaires. $.^{10} 1922-28$ The activation of the immune system after thermal ablation has never been investigated after thermal ablation of breast cancer but could possibly prevent micro metastases and local recurrences. ${ }^{14}$ 16-18 29 Moreover, none of the three techniques have been investigated in an outpatient setting in a Dutch population. ${ }^{8}$
A phase 2 screening study with all three techniques (MWA, RFA and CA) could help to select the optima thermal ablation technique and plan a future phase 3 study in which thermal ablation will be compared with breast conserving surgery (BCS) in terms of disease-free survival in patients with early-stage breast cancer.

\section{METHODS}

Aim

The overall study aim is to determine the efficacy rate in terms of complete ablation for the most promising techniques of thermal ablation (RFA, MWA or CA) in patients with early-stage breast cancer without an extensive component of DCIS ( $\leq 25 \%$ of the tumour), when these techniques are performed in a standardised study design and comparable patient population, to warrant the design of a randomised phase 3 trial comparing thermal ablation with surgery.

\section{Primary objective}

The primary objective is to investigate if thermal ablation (RFA, MWA and CA) is an effective treatment in terms of complete ablation rate, for patients with early-stage breast cancer (cT1N0M0) without an extensive component of DCIS ( $\leq 25 \%$ of the tumour) in an outpatient setting.

\section{Trial design}

This study has been designed as a multicentre open-label randomised phase 2 screening trial (the so-called 'picka-winner' design). ${ }^{30}$ The aim of a screening trial is to examine several treatments with the intention of selecting one optimal treatment for further testing in a phase 3 trial, and deselect those treatment candidates that are clearly inferior based on primary or secondary endpoints. In our study, the three potential candidates for a phase 3 study are: MWA, RFA and CA. Figure 1 shows the flow chart of the study procedures. A summary of the trial registration information can be found in table 1 .

\section{Participants}

Eligibility of all patients will be discussed in a multidisciplinary meeting, after which a patient information folder will be sent to eligible patients. Eligible patients will be informed and contacted by the treating surgeon during the first outpatient clinic visit. The investigator will elaborately inform the patient and sign the informed consent with the patient prior to randomization. If the tumour size is $\leq 2 \mathrm{~cm}$ on all imaging modalities (US, MRI and mammography), and the intraductal component is $\leq 25 \%$ of the total tumour area the patient will be included in the study (figure 1). Grade 2 tumours will be double checked by one of the involved pathologists.

Eligible for inclusion are postmenopausal women over the age of 45 with a diagnosis of early-stage invasive ductal breast cancer of no specific type (cT1N0M0) with an intraductal component of less than $25 \%$. The tumour should be visible on ultrasound and patients need sufficient 


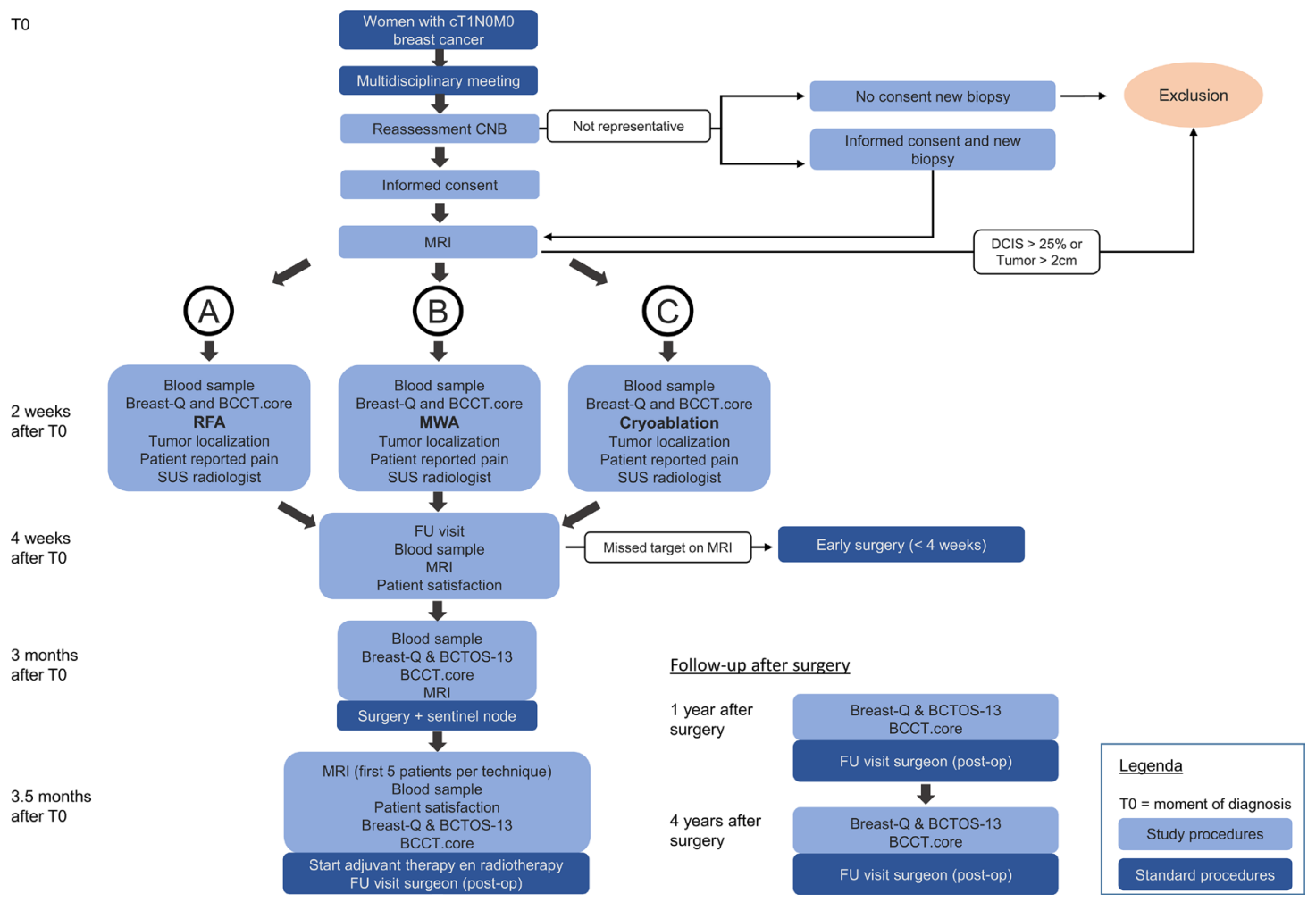

Figure 1 Flow chart study procedures. BCCT.core, Breast Cancer Conservation Treatment. cosmetic results; BCTOS, Breast Cancer Treatment Outcome Scale; CNB, core needle biopsy; DCIS, ductal carcinoma in situ; FU, follow-up; MWA, microwave ablation; RFA, radiofrequency ablation; SUS, system usability scale.

knowledge of the Dutch language to be able to complete the questionnaires. Excluded are patients with an indication for neoadjuvant chemotherapy, BRCA 1 or 2 mutations, Her2neu overexpression, triple negative tumours, and Bloom-Richardson-Elston grade 3 tumours. Patients with a history of invasive breast cancer, breast augmentation, allergy for local anaesthetics, electrical devices and/ or implants, and who are pregnant or nursing will also be excluded.

\section{Randomisation}

Patients will be allocated 1:1:1 to one of the three thermal ablation techniques (MWA, RFA or CA) using Variable Block Randomization software Ciwit B.V. (Castor EDC), with block sizes of 1, 2 or 3 . Stratification will be done by tumour size $(\leq 1 \mathrm{~cm}$ or $>1 \mathrm{~cm})$ to attempt to equally divide relative large and small tumours over the three treatment strategies. Neither patients nor physicians will be blinded since different devices and probes will be used and the physician needs to know which technique to use. The randomisation procedure, allocation and enrolment will be performed by the coordinating researcher. The assessors will be blinded for the treatment arm.

\section{Treatment groups}

Arm A: RFA with subsequent standard of care.

Arm B: MWA with subsequent standard of care.

Arm C: CA with subsequent standard of care.

\section{Intervention}

All participating physicians and supporting staff will be informed and trained prior to the start of the study. All radiofrequency, microwave and $\mathrm{CA}$ procedures will be performed in an outpatient setting by one of the three radiologists involved in the study. Two radiologists are specialised in breast cancer and experienced with tumour localisation and central needle placement, the third interventional radiologist is the proctor of radiofrequency and MWA in the Netherlands. The latter will perform at least the first five procedures together with one of the other radiologists, independent of participating centre. The combination of specialised knowledge of both disciplines will help to achieve the highest quality, reduce bias and flatten the learning curve.

All procedures will be performed under local anaesthetics (lidocaine 1\%). Light sedation can be administered if the patient prefers so or when the patient does not tolerate the pain, always with the patient's consent. Patients will be placed in supine position on the treatment table. Glucose $5 \%$ for MWA and RFA or $\mathrm{NaCl} 0.9 \%$ for $\mathrm{CA}$ will be injected between the tumour and the skin and in the retro mammary space to form an isolation belt to avoid thermal damage to the skin and pectoral muscle. A minimum of $10 \mathrm{~mm}$ distance between the tumour and the skin is recommended for safe ablation after injection of the isolation belt. A small skin incision will be made and the probe will be placed under ultrasound guidance. The tumour will be punctured along the longest axis. 
Table 1 Trial registration information

\section{Data category}

Primary registry and trial

identifying number

Date of registration in primary 20 January 2021

registry

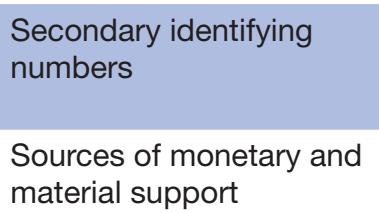

material support 2020-115

\section{Information}

Netherlands Trial Register: NL9205

Medical Research Ethics Committee: MEC-2020-0577; The Central Committee on Research Involving Human Subjects: NL72970.078.20; Franciscus Gasthuis \& Vlietland (CastorSMS):

Monetary support: Stichting BOF (Foundation for the promotion of research Franciscus); Team Westland; Stichting Coolsingel (Coolsingel Foundation); Stichting Vrienden van het Havenziekenhuis (Friends of the Havenziekenhuis Foundation); Maurits en Anna de Kock Stichting (Maurits and Anna de Kock Foundation); Material support: IceCure; AngioCare

Primary sponsor Franciscus Gasthuis \& Vlietland

Secondary sponsor(s) NA

Contact for public queries

E.M.F. van de Voort, MD, e.voort@franciscus.nl

Contact for scientific queries

Public title

Scientific title

E.M.F. van de Voort, MD, e.voort@franciscus.nl

The Treatment of Breast Cancer with Percutaneous Thermal Ablation: A phase 2 screening trial The Treatment of Breast Cancer with Percutaneous Thermal Ablation: A phase 2 screening trial (THERMAC)

Countries of recruitment The Netherlands

Health condition(s) or problem(s) studied

Intervention(s)

Key inclusion and exclusion criteria

\section{Breast cancer}

Radiofrequency ablation, microwave ablation, cryoablation

All postmenopausal women with cT1NOMO breast cancer

Key inclusion criteria: BRE grade 1 and 2, unilateral and unifocal, tumour should be visible on ultrasound, intraductal component $\leq 25 \%$ of the total tumour

Key exclusion criteria: Her2neu overexpression, genetic disposition for breast cancer, electrical devices or implants, triple negative tumours, lobular tumours, indication for neoadjuvant chemotherapy, history of breast cancer, allergy to local anaesthetics

$\begin{array}{ll}\text { Study type } & \text { Interventional } \\ & \text { Allocation: randomised comparison stratified by tumour size, three arms, open label } \\ & \text { Primary purpose: treatment } \\ & \text { Phase } 2\end{array}$

Placement of the probe will be checked in two directions and all procedures will be monitored with ultrasound. After the procedure, patients will be monitored in our hospital for at least 1-2 hours at our recovery and/or day care, depending on whether sedation was used. RFA will be performed with AMICA generator (AGN-H-1.0) equipment and corresponding $17 \mathrm{G}$ probes with an exposed tip of 30 or $35 \mathrm{~mm}$ for tumours up to $1.5 \mathrm{~cm}$ or over $1.5 \mathrm{~cm}$ respectively. AUTO mode according to the 'rolloff' principle will be used. MWA will also be performed with the AMICA generator (AGN-H-1.0) with corresponding 16G probes and a power application of 40W for 5-10 min. CA will be performed with the ProSense generator with corresponding $10 \mathrm{G}$ probes. Treatment times that will be 
used are: $9 \mathrm{~min}$ freeze (first cycle), $8 \mathrm{~min}$ thaw, $9 \mathrm{~min}$ frees (second cycle). A more detailed description of the procedures can be found in online supplemental materials 1 "Technique specific procedures".

BCS or mastectomy is performed in all patients after the allocated thermal ablation intervention. The type of surgery will be discussed with the patient prior to inclusion. In agreement with the standard of care and the Dutch guideline for the treatment of breast cancer (Mammacarcinoom 2.0), patients included in this study will most likely receive BCS. ${ }^{31}$

\section{Outcomes}

\section{Primary outcomes}

The primary outcome is the proportion of patients with complete ablation after thermal ablation as determined at pathological evaluation. The ablation zone will be measured in gross specimen in at least two dimensions, namely the smallest and largest diameter. The volume will be determined by using the following formula: $\mathrm{V}=\frac{4}{3} \Phi$ $(\mathrm{LAD} / 2)(\mathrm{SAD} / 2)^{2}$, in which $\mathrm{LAD}=$ long-axis diameter and $\mathrm{SAD}=$ short-axis diameter. Representative samples from the centre and the margin of the ablation zone and, when apparent of the tumour will be selected and embedded in paraffin. These slices will be evaluated with $\mathrm{H} \& \mathrm{E}$ and CK8/18 staining, according to the standard criteria as used for complete response after neoadjuvant chemotherapy. H\&E staining grade 1 represents no histological response, grade 2 a partial histological response, and grade 3 complete histological response (complete ablation). For CK 8/18, the following categories are being used: less than $10 \%$ (negative), $10 \%-50 \%$ and $>50 \%-$ $100 \%$. A more detailed description is provided in the online supplemental materials 2. Staining categories".

\section{Secondary outcomes}

The secondary outcomes are: feasibility of the technique in an outpatient setting, accuracy of MRI for complete ablation, patient satisfaction, adverse events (AEs), side effects, cosmetic outcome, system usability and immune response. All study procedures with corresponding time points are presented in table 2 . The feasibility of the techniques will be determined in terms of complication rate and grade according to the Clavien-Dindo classification and tolerability of the treatment measured as preliminary termination of the procedure because of pain or discomfort. The prognostic accuracy of MRI will be determined using the pathology result as the reference test. Patient satisfaction is measured in terms of pain on a visual analogue scale, overall satisfaction with a Likert scale, and two open questions (if the patient would recommend the procedure to others, and if the patient would choose thermal ablation over surgery if she would have the choice). Cosmetic outcome will be measured with the BCTOS-13, the Breast-Q questionnaire, and BCCT. core software for which photos will be taken. ${ }^{32-35}$ System usability will be measured with the system usability scale questionnaire which will be completed by the performing radiologist. ${ }^{36} \mathrm{~A}$ systemic and local immune response will be measured in blood and tissue respectively. In blood samples, a leucocyte count, lymphocyte subsets, T-cell response and cytokine profile will be measured. Tissue samples will be evaluated using Vectra analysis and RNA sequencing. Finally, all AEs, grade of fibrosis and side effects will be registered. All MRIs and specimens will be independently assessed by the three radiologists and pathologists involved in the study, respectively.

\section{Safety measures}

This study should neither lead to decreased oncological outcome nor to a significant increase in complications. Therefore, a series of stopping rules have been incorporated which will be tested after the inclusion of 5,10 and 15 patients per technique. These stopping rules are shown in table 3 . Additionally, the study will be audited by an independent monitor, and an independent data safety and monitoring board (DSMB) was installed prior to the first inclusion. Monitoring will take place according to the NFU guideline Quality Assurance Human Research 2019. The DSMB has been established in accordance with the European Medicines Agency guideline, and will perform an interim assessment of the safety data and stopping rules after inclusion of the first 10 patients per study arm. Moreover, the DSMB will review whether AEs as reported by the coordinating researcher fulfil the criteria for an event as defined in the stopping rules.

\section{Withdrawal of patients}

The investigator can decide to withdraw a subject from the study for urgent medical reasons. Subjects can withdraw from the study at any given moment for any reason if they wish to do so and will receive standard therapy from that moment onwards unless they reject surgical excision. In case consent is withdrawn before pathology results are known (ie, before the primary endpoint is reached) the subject will be replaced, unless withdrawal is due to safety or feasibility issues. Reasons for withdrawal when given by the patient will be recorded. In case of withdrawal, every effort will be made to acquire follow-up data with the patient's consent and all patients will be discouraged to skip surgical excision.

\section{Sample size}

The aim of this phase 2 study is to produce quantitative estimations of the outcome measures. This study is neither designed nor powered to demonstrate a statistically significant difference between the three treatment modalities. Based on previous literature, a complete ablation rate of $85 \%$ is considered acceptable and realistic. ${ }^{6-8}$ For this phase 2 study, we consider a $10 \%$ difference between techniques as clinically relevant. Following Sargent's and Dehbi et al methodology, 21 patients per treatment arm will be needed for this study. ${ }^{37}$ For this estimation, the following assumptions have been made: three treatment arms, randomisation ratio of 1:1:1, P most 


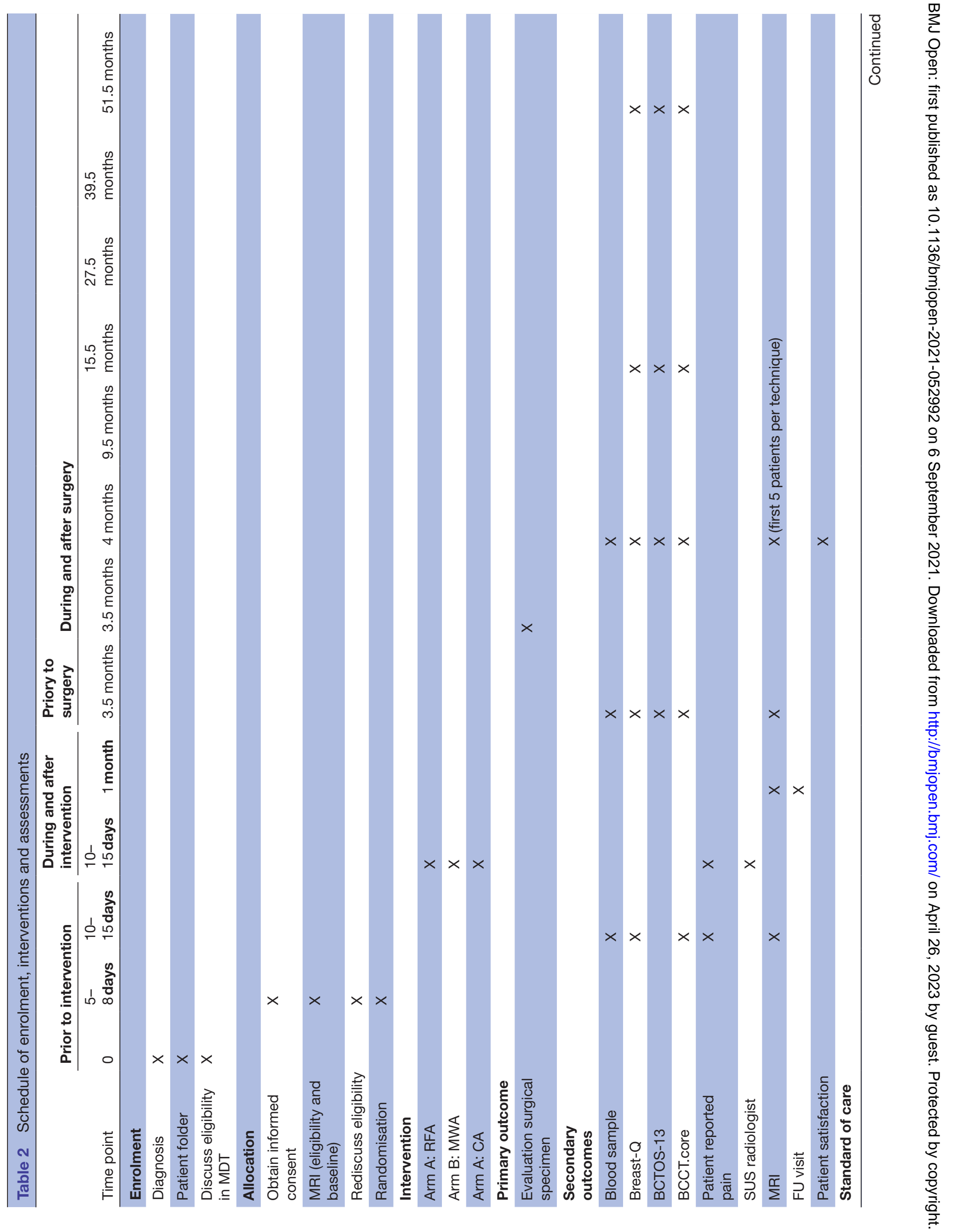




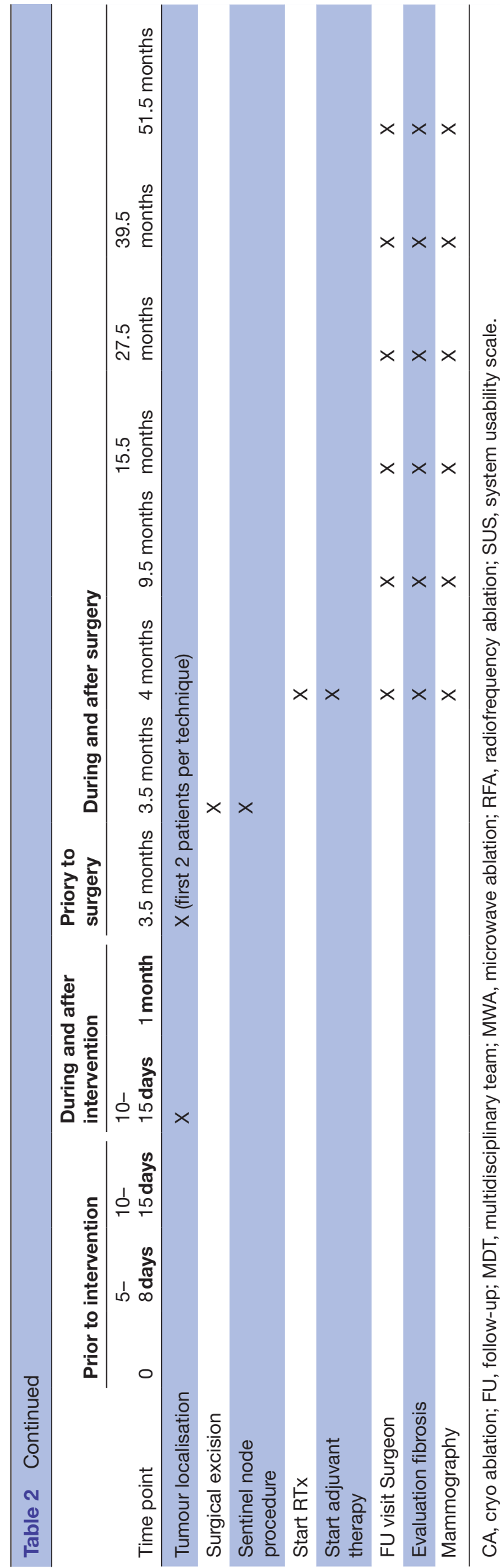

of $80 \%, \delta$ of $15 \%$, probability of success of $95 \%$, and a margin of equivalence of $5 \%$.

The Franciscus Gasthuis \& Vlietland is a large teaching hospital in which approximately 355 patients with breast cancer are treated per year. The Erasmus MC-Cancer Institute is an academic hospital in which approximately 130 patients with breast cancer with are treated per year. According to the NBCA 2018 data, approximately $60 \%$ of all patients have a cT0, cTis or cT1 tumour, $83 \%$ has a $\mathrm{cN} 0$ stage, histology shows a tumour of no special type in $75 \%$ of all cases, BRE-grade I or II occurs in $68 \%$ and a hormone positive and Her2neu negative tumour occurs in $75 \%$ of all cases. ${ }^{39}$ With these data, it is estimated that at least $20 \%$ of all patients would be eligible. An inclusion period of approximately 2 years is expected if $40 \%$ of all eligible patients would participate in the study. We anticipate a drop-out rate of $10 \%$ of all patients that sign informed consent.

\section{Statistical analysis}

Data will be analysed following intention-to-treat and per protocol. Cross-over is not expected in this study, but if cross-over appears an as-treated analysis will be performed as well. Efforts will be made to prevent missing data by recontacting subjects that did not attend their follow-up visit. Randomised groups will be compared for imbalance without formal statistical testing. Descriptive statistics include median and IQR for continuous variables, and absolute numbers with proportions for categorical variables.

Descriptive statistics will be used for the analysis of the primary and secondary outcomes. The predictive value of MRI will be expressed as prognostic accuracy with a 95\% exact CI. Differences in cosmetic outcome and immune response across the techniques over time will be analysed using a linear mixed model .

To select the optimal technique for the phase 3 study, the technique should meet the minimum requirements of an efficacy rate of at least $85 \%$, complication rate of $\leq 15 \%$, and the treatment should be completed without interruption due to pain or discomfort in at least $80 \%$ of patients. If more than one technique meets the minimum requirements and the difference in efficacy between techniques is $<5 \%$, secondary outcomes will be used to select the optimal technique for the phase 3 study. In that case, the optimal technique for the phase 3 study will be selected using predetermined rules in which the following ranking of the secondary outcomes is used: (1) positive predictive value of the MRI, (2) the proportion of complications, (3) the cosmetic outcome after thermal ablation, (4) immune response.

\section{Patient and public involvement}

The Dutch patient association for patients with breast cancer (PAG and BOOG) received a copy of the study protocol and the patient information folder. Feedback was provided by patient representatives and incorporated in these documents. The patient association has indicated 
Table 3 Stopping rules, the table describes the number of patients at which the study arm should be put on hold

\section{Stoping rules}

After 5 included

After 10 included

After 15 included

1a. Severe complications such as Clavien-Dindo grade

III complications after thermal ablation and prior to surgical resection, and/or complications related to thermal ablation which interfere with surgical excision such that the surgical procedure should be deviated (eg, surgical procedure cannot be performed due to a large haematoma).

1b. Severe complications after thermal ablation and patients per arm patients per arm patients per arm

3 patients 3 patients 4 patients
surgery, with or without radiotherapy related to the study intervention (thermal ablation), which result in unplanned mastectomy.

\begin{tabular}{l}
$\begin{array}{l}\text { 2.Premature termination of thermal ablation procedure, } \\
\text { due to pain or discomfort because of an insufficient pain } \\
\text { protocol (local anaesthetics with or without sedation). }\end{array}$ \\
$\begin{array}{l}3 \text { patients } \\
\text { 3. Irradical resection (more than focally irradical }{ }^{\star} \text { after }\end{array}$ \\
thermal ablation and surgical resection, in which, a margin \\
is considered irradical if viable or necrotic tumour tissue \\
is found in the specimen at pathologic evaluation (R1 \\
resection). \\
$\begin{array}{l}\text { 4a. Missed target (ablation zone completely outside of } \\
\text { the tumour area on MRI) on MRI } 2 \text { weeks after thermal } \\
\text { ablation. }\end{array}$ \\
$\begin{array}{l}\text { 4b. Incomplete ablation rate as determined on } \\
\text { pathological examination. }\end{array}$ \\
\hline
\end{tabular}

*An irradical resection or R1 resection is defined as viable or necrotic tumour (either invasive or DCIS) reaching in the ink in a small area $(\leq 4 \mathrm{~mm})$. The stopping rule applies to more than focally irradical tumours: viable or necrotic tumour (either invasive or DCIS) reaches the ink in a larger area $(>4 \mathrm{~mm})$ or in multiple smaller areas.

that the subject is relevant and that thermal ablation as an alternative treatment under local anaesthetics will certainly fill a need for a large group of patients because many patients are reluctant to surgery. Patients have assessed the burden of the intervention and expected sufficient willingness from patients to participate in the study. The results of this study will be communicated to the patient association, who can then disseminate the results among their members. For the development of the phase 3 trial, the patient association will be involved as well.

\section{ETHICS AND DISSEMINATION}

This study will be conducted in accordance to the standards of Good Clinical Practice, in agreement with the Declaration of Helsinki, with Dutch law in general, and with the Medical Research Involving Human Subjects Act in particular. This protocol is approved by the Medical Research Ethics Committee (METC) of the Erasmus Medical Center.

When protocol modifications are made, the new protocol has to be approved by the accredited METC before they can be implemented. Data collection, assessment and analysis will be performed according to the local guidelines for data management of the Franciscus Gasthuis \&
Vlietland hospital. Case report forms, including data validation checks and change confirmations, are created in Castor EDC prior to the start of the study.

The data of this phase 2 trial will be used to develop the protocol for the phase 3 trial to increase effectiveness and efficiency, that is, limitation of immune measurements to reduce costs and enhancement of patient inclusion criteria. The main parties that need to be involved in disseminating insights and promoting the use of thermal ablation are: physicians, nurses, hospitals, hospital managers, health insurance companies, patients with breast cancer and patient advisory groups. The results of this phase 2 trial will be presented to stakeholders during at least one national and one international scientific conference and will be submitted for publication in a peer-reviewed journal. Subsequently, the phase 3 trial will compare the most promising thermal ablation technique with BCS (non-inferiority in terms of re-excision rate and local recurrence rate). After the phase 3 trial, the results will be presented to the same parties as previously mentioned and local and national guidelines should be adjusted. Publications will target managers, healthcare professionals and researchers.

Within oneyear after study completion, the investigator/sponsor will submit a final study report with the 
results of the study, including any publications/abstracts of the study, to the accredited METC. Currently, three patients are enrolled in the study and the last patient is expected to be included in March 2023. The first results are expected to be reported in 2023.

\section{DISCUSSION}

The main objective of this trial is to determine the efficacy rate of the three most promising thermal ablation techniques with the intention of selecting one treatment for further testing in a phase 3 trial. It should be emphasised that the current phase 2 study is not designed nor powered to demonstrate a statistical significant difference between the techniques.

Although several studies previously investigated thermal ablation as a treatment for breast cancer, due to heterogeneity among studies it is unknown which of these three techniques should be recommended for a comparative phase 3 study. ${ }^{6-8}$ This is the first study in which the three most promising thermal ablation techniques will be tested according to a uniform study protocol. Unique in this phase 2 study design is the prolonged interval of 3 months between thermal ablation and surgical excision. Hence, complete ablation after thermal ablation can adequately be assessed and the risk of underestimation of the actual complete ablation rate is highly reduced. ${ }^{8202740}$ Additionally, this prolonged interval provides the opportunity to investigate the cosmetic outcome, the accuracy of MRI and the immune response after thermal ablation. ${ }^{11-1416-19}{ }^{29}$ By following up all patients up to 4 years after surgery, long-term effects of thermal ablation, surgical excision and radiotherapy can be monitored as these are not described in any of the previous studies on thermal ablation. To decrease treatment morbidity, a new technique should be feasible under local anaesthesia in an outpatient setting. By performing all techniques under standardised conditions, this will provide more insight in which technique will be most suitable in an outpatient setting.

The evaluation of complete ablation will remain an important challenge. ${ }^{41}$ If MRI can be used to select those patients in whom complete ablation is established and surgical excision can be avoided in a future phase 3 trial, will therefore be investigated in this phase 2 study. If MRI will not be sensitive enough to reliably select patients with complete ablation, other options with a better diagnostic accuracy should be considered for the phase 3 trial. For example, it is possible to perform both an MRI and (vacuum assisted) biopsies of the ablation zone to assess complete ablation.

In this protocol, a large appeal is made to patients' altruism to participate, as surgical excision is still needed. This could negatively impact the inclusion rate. However, potential benefits like immune response are investigated, and no altered prognosis is expected in this group of patients with low-risk tumours in which at least part of the tumour will be ablated within 2 weeks after diagnosis.
To further improve the safety of patients in this study, an MRI is performed 2 weeks after thermal ablation to prevent undertreatment. Surgical procedure and adjuvant therapy will be performed as soon as possible if the ablation area appears to be outside of the tumour. Additionally, stopping rules are implemented to prevent that an insufficient technique will be continued.

The exact mechanism and balance between oncogenic and immunologic effects after thermal ablation remain topic of debate in current literature. Increased vascularity as seen in the peripheral ring of the ablation zone due to inflammation might theoretically lead to a higher risk of dissemination of tumour cells. However, based on previous clinical studies, this effect is not expected after thermal ablation of primary early-stage breast cancer. Moreover, it is expected that the immune response after thermal ablation might provide protection against tumours. ${ }^{1042-44}$ More research on this topic is necessary and by measuring both the local as systemic immune response after thermal ablation this study could contribute to this knowledge gap.

In conclusion, this study could close the knowledge gap on thermal ablation for breast cancer that is apparent in current literature and will provide the necessary data to warrant a randomised phase 3 trial comparing thermal ablation with the standard of care (BCS). If this phase 3 trial is successful, thermal ablation could be an acknowledged alternative for surgical excision. Thereby, the risks and disadvantages associated with surgical excision and general anaesthesia can be avoided in a large proportion of patients with breast cancer in the future. Additionally, cosmetic outcome and the resulting health-related quality of life of these patients could be further improved.

\section{Author affiliations \\ ${ }^{1}$ Department of Surgery, Franciscus Gasthuis en Vlietland, Rotterdam, The Netherlands \\ ${ }^{2}$ Department of Surgical Oncology, Erasmus Medical Center, Rotterdam, The Netherlands \\ ${ }^{3}$ Department of Radiology and Nuclear Medicine, Erasmus Medical Center, Rotterdam, The Netherlands \\ ${ }^{4}$ Laboratory of Tumor Immunology, Department of Medical Oncology, Erasmus Medical Center, Rotterdam, The Netherlands \\ ${ }^{5}$ Department of Radiology, Franciscus Gasthuis en Vlietland, Rotterdam, The Netherlands \\ ${ }^{6}$ Department of Pathology, Pathan B.V, Rotterdam, The Netherlands ${ }^{7}$ Department of Radiotherapy, Erasmus Medical Center, Rotterdam, The Netherlands ${ }^{8}$ Department of Statistics and Education, Franciscus Gasthuis en Vlietland, Rotterdam, The Netherlands \\ ${ }^{9}$ Department of Genetics, University Medical Centre Groningen, Groningen, The Netherlands}

Acknowledgements We would like to thank the Academic Breast Cancer working group of the Erasmus MC and the Patient Advocacy Group of the Dutch Breast Cancer Association for their advice and guidance during the design of the study.

Contributors EMFvdV drafted the first version of this manuscript. All authors drafted the original study protocol and revised the manuscript. TMALK and CV supervised the drafting of the study protocol and manuscript. TMALK, GS and EMFvdV acquired funding for the trial. TMALK initiated the trial and is the principal investigator.

Funding This work was monetary supported by Team Westland, Stichting BOF (Foundation for the promotion of research Franciscus), Stichting Coolsingel 
(Coolsingel Foundation), Stichting Vrienden van het Havenziekenhuis (Friends of the Havenziekenhuis Foundation), Maurits en Anna de Kock Stichting (Maurits and Anna de Kock Foundation). Material support was provided by IceCure and AngioCare.

Disclaimer None of these funding sources had a role in the design of this study and they will not have any role during its execution, analyses, interpretation of the data, or decision to submit results.

Competing interests None declared.

Patient consent for publication Not required.

Ethics approval Ethics Committee: Medische Ethische Toetsings Commissie Erasmus MC Protocol ID: MEC-2020-0577.

Provenance and peer review Not commissioned; externally peer reviewed.

Data availability statement Data are available upon reasonable request. Deidentified individual clinical trial participant-level data, protocols and the statistical analysis plan will be available upon reasonable request at publication. These data will be available for researchers who provide a methodologically sound proposal to achieve the aims in the approved proposal. Proposals should be directed to info@stichtingbor.nl and to gain access, data requestors will need a data access agreement. This data sharing plan was updated in the trial registry (https://www.trialregister.nl/edit/9205) on 13-07-20.

Supplemental material This content has been supplied by the author(s). It has not been vetted by BMJ Publishing Group Limited (BMJ) and may not have been peer-reviewed. Any opinions or recommendations discussed are solely those of the author(s) and are not endorsed by BMJ. BMJ disclaims all liability and responsibility arising from any reliance placed on the content. Where the content includes any translated material, BMJ does not warrant the accuracy and reliability of the translations (including but not limited to local regulations, clinical guidelines, terminology, drug names and drug dosages), and is not responsible for any error and/or omissions arising from translation and adaptation or otherwise.

Open access This is an open access article distributed in accordance with the Creative Commons Attribution Non Commercial (CC BY-NC 4.0) license, which permits others to distribute, remix, adapt, build upon this work non-commercially, and license their derivative works on different terms, provided the original work is properly cited, appropriate credit is given, any changes made indicated, and the use is non-commercial. See: http://creativecommons.org/licenses/by-nc/4.0/.

\section{ORCID iD}

Elles M F van de Voort http://orcid.org/0000-0003-2392-9763

\section{REFERENCES}

1 KWF Kankerbestrijding, N. Borstkanker, 2019. Available: prof. dr. Tom Würdinger [Accessed 11 Feb 2021].

2 Integraal Kankercentrum Nederland, k.n. Overlevingscijfers borstkanker, Stadiumverdeling bij diagnose in 2015 van borstkanker. 2015 02-2018. Available: https://www.kanker.nl/kankersoorten/ borstkanker/wat-is/overlevingscijfers-borstkanker [Accessed 25 Jan 2019].

3 Team, T.A.C.S.M.a.E.C. breast cancer survival rates 201712 2018. Available: https://www.cancer.org/cancer/breast-cancer/ understanding-a-breast-cancer-diagnosis/breast-cancer-survivalrates.html [Accessed 25 Jan 2019].

4 Monticciolo DL, Helvie MA, Hendrick RE. Current issues in the overdiagnosis and overtreatment of breast cancer. AJR Am J Roentgenol 2018;210:285-91.

5 Ahmed M, Brace CL, Lee FT, et al. Principles of and advances in percutaneous ablation. Radiology 2011;258:351-69.

6 Mauri G, Sconfienza LM, Pescatori LC, et al. Technical success, technique efficacy and complications of minimally-invasive imagingguided percutaneous ablation procedures of breast cancer: a systematic review and meta-analysis. Eur Radiol 2017;27:3199-210.

7 Peek MCL, Ahmed M, Napoli A, et al. Minimally invasive ablative techniques in the treatment of breast cancer: a systematic review and meta-analysis. Int J Hyperthermia 2017;33:191-202.

8 van de Voort EMF, Struik GM, Birnie E, et al. Thermal Ablation as an Alternative for Surgical Resection of Small $(\leq 2 \mathrm{~cm})$ Breast Cancers: A Meta-Analysis. Clin Breast Cancer 2021. doi:10.1016/j. clbc.2021.03.004. [Epub ahead of print: 17 Mar 2021].

9 Kinoshita T, Yamamoto N, Fujisawa T, et al. Radiofrequency ablation for early-stage breast cancer: results from 5 years of follow-up in a prospective multicenter study. JCO 2017;35:e12098.
10 Fine R, Berry M. ICE3 trial: cryoablation of low-risk, early-stage breast cancers $1.5 \mathrm{~cm}$ : an evaluation of local recurrence-An interim update. Ann Surg Oncol 2018;25:334-5.

11 Gage AA, Baust J. Mechanisms of tissue injury in cryosurgery. Cryobiology 1998;37:171-86.

12 Chu KF, Dupuy DE. Thermal ablation of tumours: biological mechanisms and advances in therapy. Nat Rev Cancer 2014;14:199-208.

13 Gage AA. Cryosurgery in the treatment of cancer. Surg Gynecol Obstet 1992;174:73-92.

14 Mehta A, Oklu R, Sheth RA. Thermal ablative therapies and immune checkpoint modulation: can locoregional approaches effect a systemic response? Gastroenterol Res Pract 2016;2016:1-11.

15 Slovak R, Ludwig JM, Gettinger SN, et al. Immuno-thermal ablations - boosting the anticancer immune response. J Immunother Cancer 2017;5:78.

16 Takaki H, Cornelis F, Kako Y, et al. Thermal ablation and immunomodulation: from preclinical experiments to clinical trials. Diagn Interv Imaging 2017;98:651-9.

17 Jansen MC, van Hillegersberg R, Schoots IG, et al. Cryoablation induces greater inflammatory and coagulative responses than radiofrequency ablation or laser induced thermotherapy in a rat liver model. Surgery 2010;147:686-95.

18 McArthur HL, Diab A, Page DB, et al. A pilot study of preoperative single-dose ipilimumab and/or cryoablation in women with earlystage breast cancer with comprehensive immune profiling. Clin Cancer Res 2016;22:5729-37.

19 Manenti G, Scarano AL, Pistolese CA, et al. Subclinical breast cancer: minimally invasive approaches. our experience with percutaneous radiofrequency ablation vs. cryotherapy. Breast Care 2013;8:356-60.

20 Ohtani S, Kochi M, Ito M, et al. Radiofrequency ablation of early breast cancer followed by delayed surgical resection--a promising alternative to breast-conserving surgery. Breast 2011;20:431-6.

21 Simmons RM, Ballman KV, Cox C, et al. A phase II trial exploring the success of cryoablation therapy in the treatment of invasive breast carcinoma: results from ACOSOG (Alliance) Z1072. Ann Surg Oncol 2016;23:2438-45.

22 García-Tejedor A, Guma A, Soler T, et al. Radiofrequency ablation followed by surgical excision versus lumpectomy for early stage breast cancer: a randomized phase II clinical trial. Radiology 2018;289:317-24.

23 Kinoshita S, Miyake R, Shimada N, et al. Initial experience with contrast-enhanced ultrasonography in follow up assessment of small breast cancer treated by cryoablation. Australas Med J 2017;10.

24 Medina-Franco H, Soto-Germes S, Ulloa-Gómez JL, et al. Radiofrequency ablation of invasive breast carcinomas: a phase II trial. Ann Surg Oncol 2008;15:1689-95.

25 Oura S, Tamaki T, Hirai I, et al. Radiofrequency ablation therapy in patients with breast cancers two centimeters or less in size. Breast Cancer 2007;14:48-54.

26 Yamamoto N, Fujimoto $\mathrm{H}$, Nakamura R, et al. Pilot study of radiofrequency ablation therapy without surgical excision for T1 breast cancer: evaluation with MRI and vacuum-assisted core needle biopsy and safety management. Breast Cancer 2011;18:3-9.

27 Yoshinaga Y, Enomoto Y, Fujimitsu R, et al. Image and pathological changes after radiofrequency ablation of invasive breast cancer: a pilot study of nonsurgical therapy of early breast cancer. World $J$ Surg 2013;37:356-63.

28 Schwartzberg B, Lewin J, Abdelatif O, et al. Phase 2 open-label trial investigating percutaneous laser ablation for treatment of early-stage breast cancer: MRI, pathology, and outcome correlations. Ann Surg Oncol 2018;25:2958-64

29 Slovak R, Ludwig JM, Gettinger SN, et al. Immuno-thermal ablations - boosting the anticancer immune response. J Immunother Cancer 2017:5:78.

30 Simon R, Wittes RE, Ellenberg SS. Randomized phase II clinical trials. Cancer Treat Rep 1985;69:1375-81.

31 van der Sangen MJC, Obdeijn AIM, Hairwassers LJ, et al. Werkgroep richtlijn Borstkanker 2.0. Richtlijn: Borstkanker, 2020.

32 Struik GM, de Jongh FW, Birnie E, et al. Development and psychometric evaluation of a Dutch-translated shorter breast cancer treatment outcome scale (Dutch BCTOS-13). J Patient Rep Outcomes 2018;2:60.

33 Hennigs A, Heil J, Wagner A, et al. Development and psychometric validation of a shorter version of the breast cancer treatment outcome scale (BCTOS-12). Breast 2018;38:58-65.

34 Pusic AL, Klassen AF, Scott AM, et al. Development of a new patientreported outcome measure for breast surgery: the BREAST-Q. Plast Reconstr Surg 2009;124:345-53. 
35 Cardoso MJ, Cardoso JS, Wild T, et al. Comparing two objective methods for the aesthetic evaluation of breast cancer conservative treatment. Breast Cancer Res Treat 2009;116:149-52.

36 Bangor A, Kortum PT, Miller JT. An empirical evaluation of the system usability scale. Int J Hum Comput Interact 2008;24:574-94.

37 Sargent DJ, Goldberg RM. A flexible design for multiple armed screening trials. Stat Med 2001;20:1051-60.

38 Dehbi H-M, Hackshaw A. Sample size calculation in randomised phase II selection trials using a margin of practical equivalence. Trials 2020;21:301.

39 NABON D. IKNL. Jaarverslag NBCA, 2018. Available: https://dica.nl/ media/2234/NBCA\%20jaarverslag\%202018.pdf [Accessed 24 Jul 2020].

40 Motoyoshi A, Noguchi M, Earashi M, et al. Histopathological and immunohistochemical evaluations of breast cancer treated with radiofrequency ablation. J Surg Oncol 2010;102:385-91.
41 van de Voort EMF, Struik GM, Birnie E. Thermal ablation as an alternative for surgical resection of small $(\leq 2 \mathrm{~cm})$ breast cancers: a meta-analysis. Clin Breast Cancer 2021;17.

42 Kinoshita T, Takahashi M, Fujisawa T, et al. Radiofrequency ablation therapy for early-stage breast cancer: results from 5 years of follow-up in a prospective multicenter study. Eur $J$ Cancer 2018;92:S63.

43 Zhou W, Yu M, Pan H, et al. Microwave ablation induces Th1-type immune response with activation of ICOS pathway in early-stage breast cancer. J Immunother Cancer 2021;9:e002343.

$44 \mathrm{Yu} \mathrm{M}, \mathrm{Pan} \mathrm{H}$, Che N, et al. Microwave ablation of primary breast cancer inhibits metastatic progression in model mice via activation of natural killer cells. Cell Mol Immunol 2020. doi:10.1038/s41423-0200449-0. [Epub ahead of print: 08 May 2020]. 